\title{
Cerebral Palsy and Stroke-Early and Late Brain Lesion Present Differences in Systemic Biomarkers and Gene Expression Related to Muscle Contractures
}

\author{
Jessica Pingel ${ }^{1}$, Camille Potts ${ }^{1}$, Theis Wolter Petersen${ }^{1}$, Jens Bo Nielsen ${ }^{1,2}$ \\ ${ }^{1}$ Department of Neuroscience, University of Copenhagen, Copenhagen, Denmark \\ ${ }^{2}$ The Elsass Foundation, Research Unit, Charlottenlund, Denmark \\ Email: *jpingel@sund.ku.dk
}

How to cite this paper: Pingel, J., Potts, C., Petersen, T.W. and Nielsen, J.B. (2021) Cerebral Palsy and Stroke-Early and Late Brain Lesion Present Differences in Systemic Biomarkers and Gene Expression Related to Muscle Contractures. World Journal of Neuroscience, 11, 34-47. https://doi.org/ 10.4236/wjns.2021.111005

Received: November 14, 2020

Accepted: February 22, 2021

Published: February 25, 2021

Copyright $\odot 2021$ by author(s) and Scientific Research Publishing Inc. This work is licensed under the Creative Commons Attribution International License (CC BY 4.0).

http://creativecommons.org/licenses/by/4.0/

\begin{abstract}
Background: CNS lesions that are acquired early in life e.g. cerebral palsy (CP) disturb muscle development and growth, while CNS injuries acquired later in life e.g. stroke, affect fully matured muscles and cause paresis and atrophy. These differences may result in different contracture phenotypes. Aim: The purpose of this study was to compare systemic biomarkers and gene expression levels in muscle of individuals with CNS lesions acquired early and later in life. Methods: Blood samples and muscle biopsies were analyzed using Enzyme-linked immunosorbent assay and Real-time PCR from $\mathrm{n}=24$ control participants, $\mathrm{n}=14$ individuals with cerebral palsy, and $\mathrm{n}$ $=12$ stroke survivors. Results: Systemic markers: Myostatin was significantly decreased in both the cerebral palsy $(\mathrm{p}=0.0051)$, and the stroke group ( $\mathrm{p}=$ 0.036). Creatine Kinase-MB and C-Reactive Protein were significantly elevated in stroke patients only ( $p<0.007 \& \mathrm{p}>0.034$ respectively). Gene expressions: The expression of myostatin (MSTN) was significantly lower in both the ST and the $\mathrm{CP}$ group when compared to Ctrl $(\mathrm{p}=0.02)$. In addition, collagen type 4A1 (COL4A1) was significantly lower in the CP group compared to the other groups $(\mathrm{p}=0.015)$. Finally, the troponin 1 slow skeletal muscle type was significantly increased in the ST group when compared to both $\mathrm{CP}$ and Ctrl $(\mathrm{p}=0.03)$. Conclusion: The downregulation of myostatin in individuals with both early and late CNS injury is likely a compensatory reaction to muscle weakness, reduced muscle mass and/or muscle atrophy. Changes in gene expression may reflect a specific alteration depending on when in life the CNS lesions were acquired.
\end{abstract}




\section{Keywords}

Cerebral Palsy, Stroke, Biomarkers, Creatin Kinase, Myostatin, C-Reactive Protein

\section{Introduction}

Muscle contractures are common complications following lesions to the central nervous system regardless of whether the lesion occurs early in life (e.g. Cerebral palsy; [1]) or late in life (e.g. stroke; [2]). Muscle contractures are characterized by increased resistance of the passive elastic elements in the muscle and surrounding tissue leading to reduced range of movement and dislocation of joints [3]. Muscle contractures are therefore one of the most debilitating complications to central nervous lesion causing pain, reduced mobility, compromised hygiene and reduced social participation [4]. There is at present no effective therapy of muscle contractures, although considerable efforts are put into stretching and other forms of physical therapy [5]. Part of the reason why we have not been successful in finding an effective therapy is that the pathophysiology of contractures has not been clarified. There is increasing evidence to suggest that reduced growth of muscles in infants with cerebral palsy may lead to excessive stress on the muscles as bones grow longer and thereby trigger the development of contractures [6] [7]. However, this cannot explain the development of contractures in adults following stroke and other lesions of the central nervous system. It has been assumed for decades that increased muscle activity due to spasticity maintains muscles in a shortened position resulting in development of contractures. However, several studies have now documented that altered resistance of the passive elastic elements in the muscle occurs prior to development of spasticity and that contractures still develop when spasticity has been effectively abolished by section of dorsal roots [8].

We have recently argued that the lack of a pathophysiological explanation of contractures may reflect that the pathophysiology is complex and likely involves multiple factors in a complex network of tissues, stimuli, and regulatory factors that define tissue homeostasis [3]. Comparing these factors in individuals with cerebral palsy and stroke provides an opportunity to explore which of these factors are similarly altered following lesions early and late in life. Identifying common patterns of changes would help to clarify whether similar signaling pathways or separate pathophysiological mechanisms are involved when lesions occur early and late in life. The present study is a first attempt at addressing this issue by comparing systemic and gene expression levels of specific factors related to contracture development in individuals with cerebral palsy or stroke

\section{Materials \& Methods}

All participants were Danish citizens, recruited in Copenhagen Denmark and 
gave informed consent. The protocol was approved by the Regional Ethics Committee for Copenhagen (H-4-2014-047). The protocol was in compliance with the Helsinki Declaration. Blood samples were taken from healthy volunteers, individuals with $\mathrm{CP}$, and stroke survivors. All but one of the ELISA studies included 50 participants: 24 healthy $(\mathrm{n}=24 \mathrm{Ctrl}), 14$ with spastic CP $(\mathrm{n}=14$; $\mathrm{CP})$, and 12 stroke survivors $(\mathrm{n}=12$; $\mathrm{ST})$. All subject characteristics are shown in Table 1 in Mean \pm SD. The Inclusion criteria for the Ctrl group were both female and male subjects above 18 years of age with no prior history of muscle disease or any other chronic diseases. The exclusion criteria of the Ctrl group were less than 18 years of age, chronic diseases and prior muscle disease. The Inclusion criteria for the $\mathrm{CP}$ group were: individuals with spastic CP (both female and male) above 18 years of age with muscle contractures in at least one joint. In Denmark most individuals with $\mathrm{CP}$ are diagnosed with $\mathrm{CP}$ with less than two years of age.

The exclusion criteria of the $\mathrm{CP}$ group were less than 18 years of age and lack of contractures.

The Inclusion criteria for the ST group were: individuals above 18 years of age (both female and male), who had experienced a stroke and suffered from muscle contractures in at least one joint. The exclusion criteria of the ST group was less than 18 years of age and lack of contractures. The included participants had a Mean age of: $42 \pm 3$ in the CP group and $47 \pm 3$ in the ST group. The gender distribution was as follows: (CP group $n=8$ females and $n=6$ males); (ST group $n$ $=6$ females and $\mathrm{n}=6$ males). Thus, individuals from the $\mathrm{CP}$ and ST group also underwent a clinical investigation were the Modified Ashworth scale (MAS) and the range of motion (ROM) in their ankle joints was measured and recorded (Table 2). Type of CP and a score according to the Gross Motor Function Classification system (GMFCS) were recorded. The clinical history of the participants from the CP and the ST group is shown in detail in Table 2. All blood samples were taken from the medial cubital vein and centrifuged to separate the plasma. The plasma was kept at $-80^{\circ} \mathrm{C}$ until testing. The systemic levels of all markers in the plasma were determined through enzyme-linked immunosorbent assay (ELISA).

Collagen IV levels were detected using a Sandwich ELISA (Human Collagen IV ELISA Kit from LifeSpan BioSciences, Inc. Seattle, WA), with analytic sensitivity $7.8-500 \mathrm{ng} / \mathrm{mL}$ and intra-inter assay $\mathrm{CV}$ of $<10 \% \&<12 \%$ respectively.

Table 1. Subject characteristics.

\begin{tabular}{|c|c|c|c|c|c|c|}
\hline Group & Age (y) & Height (m) & Weight (kg) & $\begin{array}{c}\text { BMI } \\
\text { (weight/height }{ }^{2} \text { ) }\end{array}$ & $\begin{array}{l}\text { Time since } \\
\text { stroke }(y)\end{array}$ & Gender F/M \\
\hline $\mathrm{CP}$ & $41.9 \pm 12$ & $173.3 \pm 11$ & $71.9 \pm 13$ & $24.3 \pm 7$ & & 8 female $/ 6$ male \\
\hline ST & $46.6 \pm 11$ & $176.1 \pm 8$ & $74.8 \pm 13$ & $24.0 \pm 3$ & $3.6 \pm 1.3$ & 6 female $/ 6$ male \\
\hline Ctrl & $40.5 \pm 9$ & $177 \pm 11$ & $80.5 \pm 14$ & $25.8 \pm 4$ & & 9 female $/ 4$ male \\
\hline
\end{tabular}

All data are shown in Mean \pm SD 
Table 2. Clinical history.

\begin{tabular}{|c|c|c|c|c|c|c|c|c|c|c|}
\hline & Diagnosis & Type & Walking Aids & Sex & Age & GMFCS & $\begin{array}{c}\text { MAS } \\
\text { plantarflexion } \\
\text { (right) }\end{array}$ & $\begin{array}{c}\text { MAS } \\
\text { plantarflexion } \\
\text { (left) }\end{array}$ & $\begin{array}{c}\text { ROM } \\
\text { dorsiflexion } \\
\text { (right) }\end{array}$ & $\begin{array}{c}\text { ROM } \\
\text { dorsiflexion } \\
\quad(\text { left })\end{array}$ \\
\hline \multicolumn{11}{|l|}{$\mathrm{CP}$} \\
\hline $\mathrm{CP} 1$ & Spastic CP & diplegic & Crutches & M & 25 & 3 & 1 & 1 & 20 & 20 \\
\hline $\mathrm{CP} 2$ & Spastic CP & diplegic & Crutches & $\mathrm{F}$ & 56 & 2 & 1 & 3 & 20 & 10 \\
\hline $\mathrm{CP} 3$ & Spastic CP & hemiplegic & - & M & 42 & 1 & 0 & 1 & 20 & 10 \\
\hline $\mathrm{CP} 4$ & Spastic CP & quadriplegic & Wheelchair & $\mathrm{F}$ & 49 & 3 & 0 & 1 & 10 & 10 \\
\hline CP5 & Spastic CP & quadriplegic & Wheelchair & M & 24 & 4 & 2 & 1 & 10 & 10 \\
\hline $\mathrm{CP} 6$ & Spastic CP & diplegic & Crutches & $\mathrm{F}$ & 48 & 2 & 2 & 0 & 20 & 20 \\
\hline $\mathrm{CP} 7$ & Spastic CP & diplegic & Walking stick & $\mathrm{F}$ & 48 & 2 & 4 & 2 & 0 & 10 \\
\hline $\mathrm{CP} 8$ & Spastic CP & diplegic & Wheelchair & $\mathrm{F}$ & 51 & 2 & 3 & 2 & 10 & 0 \\
\hline СР9 & Spastic CP & diplegic & Crutches & $\mathrm{F}$ & 58 & 3 & 1 & 1 & 20 & 20 \\
\hline $\mathrm{CP} 10$ & Spastic CP & diplegic & Crutches & M & 49 & 2 & 0 & 0 & 20 & 20 \\
\hline CP11 & Spastic CP & hemiplegic & - & M & 48 & 2 & 2 & 3 & 10 & 0 \\
\hline $\mathrm{CP} 12$ & Spastic CP & diplegic & Wheelchair & $\mathrm{F}$ & 38 & 3 & 0 & 0 & 0 & 10 \\
\hline $\mathrm{CP} 13$ & Spastic CP & diplegic & Crutches & M & 25 & 1 & 0 & 0 & 10 & 10 \\
\hline $\mathrm{CP} 14$ & Spastic CP & diplegic & Crutches & $\mathrm{F}$ & 25 & 3 & 2 & 0 & 10 & 20 \\
\hline \multicolumn{11}{|l|}{ Stroke } \\
\hline ST1 & $\begin{array}{l}\text { Brain tumor } \\
\text { and stroke }\end{array}$ & hemiplegic & Orthosis & $\mathrm{F}$ & 34 & - & 3 & 0 & 5 & 22 \\
\hline ST2 & Stroke & hemiplegic & Orthosis & $\mathrm{F}$ & 57 & - & 0 & 0 & 20 & 0 \\
\hline ST3 & $\begin{array}{c}\text { Stroke after } \\
\text { accident }\end{array}$ & hemiplegic & Orthosis & $\mathrm{M}$ & 46 & - & 0 & 2 & 15 & 0 \\
\hline ST4 & Stroke & hemiplegic & Orthosis & $\mathrm{F}$ & 24 & - & 3 & 0 & -5 & 25 \\
\hline ST5 & Stroke & hemiplegic & Orthosis & $\mathrm{F}$ & 48 & - & 0 & 3 & 20 & 20 \\
\hline ST6 & Stroke & hemiplegic & Scooter & M & 52 & - & 3 & 0 & -10 & 20 \\
\hline ST7 & $\begin{array}{l}\text { Stroke after } \\
\text { accident }\end{array}$ & hemiplegic & Orthosis & M & 48 & - & 0 & 3 & 10 & 0 \\
\hline ST8 & Stroke & hemiplegic & Orthosis & $\mathrm{F}$ & 45 & - & 0 & 2 & 20 & 20 \\
\hline ST9 & Stroke & hemiplegic & Orthosis & $\mathrm{F}$ & 55 & - & 0 & 3 & 25 & 0 \\
\hline ST10 & Stroke & hemiplegic & Orthosis & M & 48 & - & 3 & 0 & 0 & 25 \\
\hline ST11 & Stroke & hemiplegic & Orthosis & M & 38 & - & 2 & 0 & 10 & 25 \\
\hline ST12 & Stroke & hemiplegic & Orthosis & M & 65 & - & 0 & 1 & 10 & 15 \\
\hline
\end{tabular}

Troponin I levels were detected using a sandwich ELISA (Human Troponin I from Sigma-aldrich, Saint Louis, MO. USA), with a min. detectable dose of 0.61 $\mathrm{ng} / \mathrm{mL}$ and intra-inter assay $\mathrm{CV}$ of $<10 \% \&<12 \%$ respectively.

Matrix Metalloproteinase-2 (MMP-2) levels were detected using a sandwich 
ELISA (Human Troponin I from Sigma-aldrich, Saint Louis, MO. USA), with a min. detectable dose of $3.5 \mathrm{pg} / \mathrm{mL}$ and intra-inter assay $\mathrm{CV}$ of $<10 \% \&<12 \%$ respectively.

Myostatin levels were detected using a sandwich ELISA (Quantikine ELISA for GDF-8/Myostatin), with a min. detectable dose of $2.25 \mathrm{pg} / \mathrm{mL}$ and intra-inter assay $\mathrm{CV}$ of $<4 \% \&<5 \%$ respectively.

Creatine Kinase-MB (CKMB) levels were detected using a sandwich ELISA (Human CKMB ELISA Kit from ThermoScientific), with a min. detectable dose of $0.3 \mathrm{ng} / \mathrm{mL}$ and intra-inter assay $\mathrm{CV}$ of $<10 \% \&<12 \%$ respectively.

C-Reactive Protein (CRP) levels were detected using a sandwich ELISA (Human C-Reactive Protein from $\mathrm{R} \& \mathrm{D}$ Systems), with a min. detectable dose of $0.01 \mathrm{ng} / \mathrm{mL}$ and intra-inter assay $\mathrm{CV}$ of $<6 \% \&<7 \%$ respectively.

\section{Muscle biopsy procedure}

The muscle biopsies were obtained from the medial Gastrochnemius muscle after local anesthesia (1\% lidocaine) and incision of the overlying skin. The biopsy was taken using a 5-mm Bergstrom needle (Stille, Stockholm, Sweden) with manual suction. The obtained muscle biopsies weighted around $80-100 \mathrm{mg}$. The samples were snapfrozen in liquid nitrogen and stored at $-80^{\circ} \mathrm{C}$ until further investigation.

\section{RNA extraction}

Total RNA was extracted from fresh frozen muscle samples from 24 subjects using $1 \mathrm{ml}$ of TriReagent (MRC) containing four stainless steel balls of $2.3 \mathrm{~mm}$ diameter (BioSpec Products, Inc., Bartlesville, Oklahoma, USA), and one silicon-carbide sharp particle of $1 \mathrm{~mm}$ (BioSpec Products, Inc.), by shaking in a Precellys Evolution tissue homogenizer (Bertin Instruments, Rockville, USA) at maximal speed for $15 \mathrm{~s}$. In order to obtain complete homogenization of the tissue, the shaking process was repeated four times with cooling on ice between each shaking step (to avoid heating of the sample). Following homogenization, bromo-chloropropane was added to separate the samples into an aqueous and an organic phase. Following isolation of the aqueous phase, RNA was precipitated using isopropanol. The RNA pellet was washed in 75\% ethanol and subsequently dissolved in RNase-free water. The RNA quality was verified using custom made agarose gels and the RNA concentration was determined using a Qubit 3.0 fluorometer (Invitrogen, Thermo Fisher Scientific, Eugene, Oregon, USA). The RNA samples were stored frozen at $-80^{\circ} \mathrm{C}$ until subsequent cDNA synthesis. All analysis was conducted according to the manufacturers protocols.

\section{PCR protocol}

The samples were subsequently prepared for PCR analysis using the Brilliant III ultra-Fast QPCR Master Mix kit (Agilent technologies Inc., Foster City, California, USA). In brief, for a final concentration of $300 \mathrm{nM}$ in the reaction the samples were diluted 1:500 as recommended by the manufacturers protocol and $15 \mathrm{ul}$ of mastermix dye was mixed with 0.075 ul primer per well ( $0.3 \mathrm{uM}$ forward and reverse primer pr well) (Table 3). Subsequently, each well was then filled with $23 \mathrm{ul}$ of mastermix. After that $2 \mathrm{ul}$ of prediluted cDNA was added to each 
Table 3. Primer sequences.

\begin{tabular}{cccc}
\hline Target & Alias & Sense & Antisense \\
\hline Glyceraldehyde-3-phosphate dehydrogenase & GAPDH & AGTCAGCCGCATCTTCTTTT & CATGGGTGGAATCATATTGG \\
60s acidic ribosomal protein P0 & RPLP0 & GGAAACTCTGCATTCTCGCTTCC & CCAGGACTCGITGTACCCGGTT \\
Myostatin & MSTN & TGCTGTAACCTTCCCAGGACCA & GCTCATCACAGTCAAGACCAAAATC \\
Collagen type 4A1 & COL-4A1 & TCGCTGTGGATCGGCTACTCT & CGATGAATGGCGCACTTCTAAAC \\
Collagen type 4As & COL-4A3 & ATCGTGGTCCACCAGGCTCA & CAGGGTGGCCCATAGACCCTT \\
Matrix Metallopeptidase-2 & MMP2 & CCGCCTTAACTGGAGCAAAAACA & TTGGGGAAGCCAGGATCCATT \\
Troponin 11, slow skeletal muscle type & TINNI-1 & GAGGTGGGTGACTGGAGGAAGAA & GGCACAGGGCTGGAGGAAAGGT \\
Troponin 12, fast skeletal muscle type & TINNI-2 & GCCAAGAAAAAGAAGGTGCTGTCC & TCTGCTGTITCAGCTTCGCCATC \\
\hline
\end{tabular}

well (The cDNA was pre-diluted in order to equalize the amount of cDNA to 1.5 ug in each sample).

The PCR plate was the placed in an Agilent AriaMX machine and the standard thermal protocol for the Agilent AriaMX was applied: 1 cycle for 3 min at $95^{\circ} \mathrm{C}, 40$ cycles for $5 \mathrm{sec}$ at $95^{\circ} \mathrm{C}$ followed by $10 \mathrm{sec}$ at $60^{\circ} \mathrm{C}$. Once the PCR protocol had run, the $\Delta \mathrm{R}$ values for each target were exported to Microsoft exel using the AriaMX software from Agilent Technologies. The fluorescence threshold was set at 500 for all targets. After that all raw data were normalized to the housekeeping gene GAPDH.

\section{Statistics}

Subject characteristics are shown in Mean \pm SD in Table 1. A nonparametric, unpaired Wilcoxon test was used to compare the groups and determine significant differences between the three groups. Values outside of the sensitivity range of the ELISA kits were removed from the data, as were outliers greater than two standard deviations away from the mean. All graphs and statistical tests were created and performed in $\mathrm{R}$. The level of significance was $\mathrm{p}<0.05$ for all analyses. The effect size was calculated using Cohen's $d$ effect size calculation equation. The $\mathrm{p}$ values were interpreted as follows: A P value around 0.05 was recognized as "weak evidence", while a $\mathrm{p}$ value less than 0.01 was recognized as "strong evidence" and a p value less than 0.001 was recognized as "very strong evidence".

\section{Results}

Myostatin: The ST and the CP group had significantly lower plasma myostatin levels than the Ctrl group ( $\mathrm{p}=0.036, \mathrm{p}=0.0051$ respectively, Figure 1$)$. There was no significant difference between ST and CP ( $p=0.83)$. (Mean \pm SD: $\mathrm{CP}=43.3 \pm 10.9 ; \mathrm{ST} 48.5 \mathrm{pg} / \mathrm{mL} \pm 13.8 \mathrm{pg} / \mathrm{mL} ; \mathrm{Ctrl}=7.5 \pm 30.4 \mathrm{pg} / \mathrm{mL})$.

Creatine Kinase (MB isoform) (CKMB): The CKMB levels were significantly higher in the ST group than the Ctrl group $(\mathrm{p}<0.007$, Figure 2$)$. There was no significant difference between ST and CP $(\mathrm{p}=0.26)$. Furthermore, there was no significant difference between CP and Ctrl $(\mathrm{p}=0.11)$. CKMB: Mean \pm SD: $\mathrm{CP}$ $=10.5 \pm 4.9 \mathrm{ng} / \mathrm{mL} ; \mathrm{ST}=12.8 \pm 3.3 \mathrm{ng} / \mathrm{mL} ; \mathrm{Ctrl}=8.0 \pm 3.3 \mathrm{ng} / \mathrm{mL}$. 


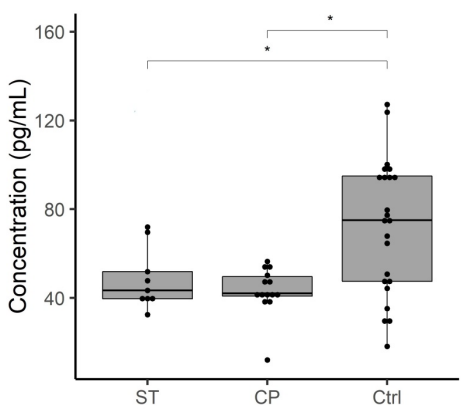

Figure 1. Myostatin plotted relative to the conditions of cerebral palsy (CP), stroke (ST), or healthy (Ctrl). The boxplots show the mean and interquartile ranges of the systemic plasma concentrations. Included observations ST $n=9$, CP $n=14$, Ctrl $n=23$. Subsequent samples were removed from the analysis because they were outside of the detection limits. Outliers beyond two standard deviations from the mean were removed. The levels of significance are $(\mathrm{p}<0.05) .{ }^{*}$ indicates significance.

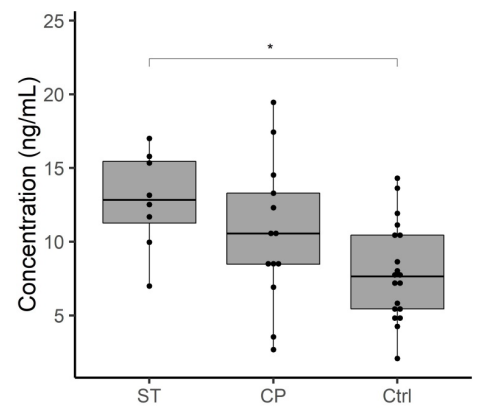

Figure 2. CKMB levels plotted relative to the conditions of cerebral palsy (CP), stroke (ST), or healthy (Ctrl). The boxplots show the mean and interquartile ranges of the systemic plasma concentrations. Included observations ST $n=8, C P n=13$, Ctrl $n=19$. Subsequent samples were removed from the analysis because they were outside of the detection limits. Outliers beyond two standard deviations from the mean were removed. The levels of significance are $(\mathrm{p}<0.05){ }^{*}$ indicates significance.

C-reactive protein (CRP): The CRP level in the ST group was significantly higher than both the CP group ( $p>0.034)$ and the Ctrl group ( $p>0.031$, Figure 3). Furthermore, there was no significant difference between $\mathrm{CP}$ and $\mathrm{Ctrl}(\mathrm{p}=$ 0.86). CRP: Mean \pm SD: $\mathrm{CP}=476.9 \pm 383.7 \mathrm{ng} / \mathrm{mL}$; ST $=1026.6 \pm 776.6 \mathrm{ng} / \mathrm{mL}$; $\mathrm{Ctrl}=471.5 \pm 350.0 \mathrm{ng} / \mathrm{mL}$.

Subsequent biomarkers: Levels of Collagen IV, Human Troponin I, and Human MMP-2 did not differ significantly ( $\mathrm{p}$ value $>0.05$ ) between CP, ST and Ctrl; means, standard deviations, and p-values are shown in Table 4.

Gene expressions: The expression of myostatin (MSTN) was significantly lower in both the ST and the CP group when compared to Ctrl $(\mathrm{p}=0.02)$. In addition collagen type 4A1 (COL4A1) was significantly lower in the $\mathrm{CP}$ group compared to the other groups $(\mathrm{p}=0.015)$. Finally the troponin 1 slow skeletal muscle type was significantly increased in the ST group when compared to both $\mathrm{CP}$ and $\mathrm{Ctrl}(\mathrm{p}=0.03)$. All subsequent gene targets showed no significant differences between any of the groups: GAPDH ( $p=0.73)$, RPLP0 ( $p=0.86)$, IL-6 $(\mathrm{p}=0.45)$, COL4A3 $(\mathrm{p}=0.09)$, TINNI-2 $(\mathrm{p}=0.28)$. 


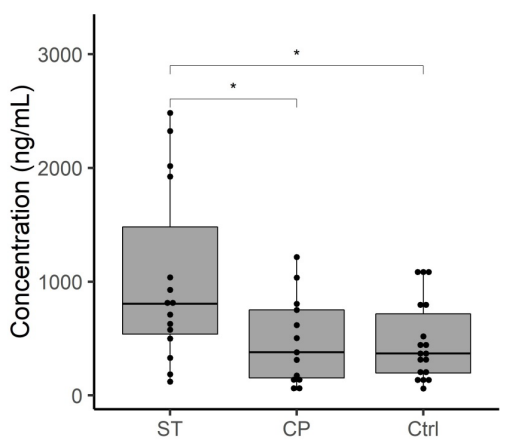

Figure 3. Levels of CRP plotted relative to the conditions of cerebral palsy (CP), stroke (ST), or healthy (Ctrl). The boxplots show the mean and interquartile ranges of the systemic plasma concentrations. Included observations ST $n=12, \operatorname{CP} n=13, \operatorname{Ctrl} n=18$. Subsequent samples were removed from the analysis because they were outside of the detection limits. Outliers beyond two standard deviations from the mean were removed. The levels of significance are $(\mathrm{p}<0.05)$. ${ }^{\star}$ indicates significance.

Table 4. Non significant biomarkers.

\begin{tabular}{cccccccccc}
\hline & \multicolumn{3}{c}{$\begin{array}{c}\text { Mean Concentration } \\
\text { (ng/mL) }\end{array}$} & & SD & \multicolumn{4}{c}{ p-values } \\
\hline Marker & ST & CP & Ctrl & ST & CP & Ctrl & ST-CP & ST-Ctrl CP-Ctrl \\
\hline Collagen IV & 26.3 & 20.6 & 11.5 & 26.2 & 9.3 & 2.2 & 0.75 & 0.77 & 0.11 \\
Human MMP-2 & 44.5 & 46.2 & 45.4 & 14.8 & 16.1 & 37.2 & 0.85 & 0.97 & 0.97 \\
Human Troponin I & 5.1 & 1.2 & 2.4 & 5.3 & n/a & 0.4 & 0.54 & 1 & 0.37 \\
\hline
\end{tabular}

The mean concentrations, standard deviations, and p-value comparisons for the markers in which no significant differences were observed between the patient groups.

\section{Discussion}

This study has revealed similarities and differences in the systemic and muscle gene expression levels of factors that are putatively involved in the pathophysiology of muscle contractures in individuals with cerebral palsy or stroke.

It is not surprising that significantly lower myostatin levels were observed in individuals with $\mathrm{CP}$ and in stroke survivors when compared to healthy controls both at the systemic level and at gene expression level in skeletal muscle (Figure 1, Figure 4). Myostatin is a growth and differentiation factor predominantly found in skeletal muscle, which greatly impacts muscle growth and function [9]. Myostatin's role as a muscle suppressor has been found across species. In humans the crucial role of Myostatin has been verified when a myostatin mutation was identified in a human child leading to abnormal muscle hypertrophy [10]. Myostatin also shows some promise as a potential therapy for conditions associated with muscle wasting and weakness [10]. Individuals with $\mathrm{CP}$ and stroke survivors have in common that they have significantly less muscle mass in their affected limbs as compared to healthy controls [11] [12]. The low levels of myostatin in individuals with $\mathrm{CP}$ and stroke may indicate a compensatory reaction to muscle weakness, reduced muscle growth and/or atrophy and increased fatigue that these patients experience [13] [14]. While muscle atrophy with 


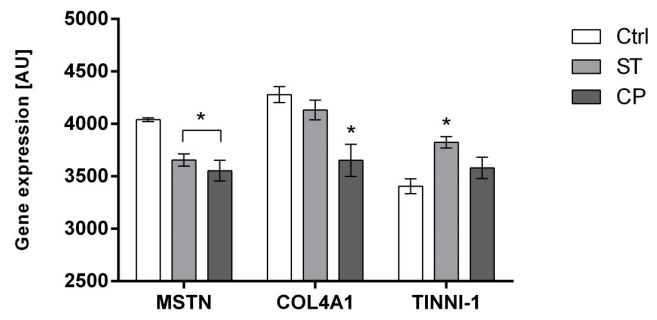

Figure 4. The normalized gene expression levels of myostatin, collagen type 4A1 and troponin-1 [AU]. Included observations: ST $n=12, \mathrm{CP} n=14, \mathrm{Ctrl} n=24$. The levels of significance is $(\mathrm{p}<0.05) .{ }^{*}$ indicates significance.

certainty is causing lower muscle mass in stroke survivors [15], there is considerable more discussion regarding the underlying mechanisms in individuals with CP due to the more complex maturation processes [6] [16] [17] [18] [19]. However, the present finding indicates that myostatin levels decrease regardless of at which stage of life an injury of the CNS is acquired.

The gene expression of collagen type 4A1 (Figure 4) was significantly lower expressed in the muscle of individuals with $\mathrm{CP}$ when compared to healthy Ctrl and stroke survivors. In contrast, no significant changes in collagen type 4 were found at the systemic level. Collagen type 4 is a crucial part of the basal membrane of muscles and the brain and has been shown to play an important role in several diseases including HANAC syndrome [20] and Walker-Warburg syndrome [21] causing various musculoskeletal symptoms including muscle weakness, hypotonia, and severe myopathy [22] [23] [24] [25]. One study showed an association between the COL4A1 gene and CP when comparing 351 individuals with $\mathrm{CP}$ with 220 healthy individuals indicating a potential role of COL4A1 in the pathogenesis of CP [26]. Altered COL4A1 DNA variants have also shown to cause diffuse or periventricular leukoen-cephalopathy [27]. In addition, COL4A1 variants were found in two atypical periventricular hemorrhagic infarction patients who were also diagnosed with spastic unilateral CP [28]. It has been suggested previously that the ubiquitously expression of COL4A1 in the body very likely is involved in the etiology of CP [26]. Since the COL4A1 expression only is affected in CNS injuries with an early onset the present findings support this notion.

$\mathrm{CKMB}$ is an intracellular enzyme found in the myocardium; therefore, high levels of CKMB in the serum suggest injury to the myocardial cell wall [29]. A previous study found elevated levels of CKMB in stroke survivors within 72 hours of onset of an acute ischemic stroke [30]. The elevated levels of CKMB in stroke are suspected to be related to cardiac myocytolysis caused by the activation of the sympathetic nervous system [31] or an increase in systemic catecholamines [32]. In the present study we observed increased CKMB levels in stroke patients when compared to healthy individuals even though the stroke of included subjects was several years prior to the present study. This suggests that the pathological effects of an CNS injury that is acquired later in life are rather long-lasting or that those patients have a higher risk for a subsequent myocardial 
infarct. Even though CKMB is mostly used as a biomarker for myocardial infarction, CKMB has also been used as a marker for muscle damage since the plasma levels of CKMB have been shown to increase during high intensity exercise [33] [34], rhabdomyolysis [35] and muscle trauma [36]. Whether muscle atrophy and contracture development might cause muscle damage, and thus affect plasma CKMB levels is unclear. However, since we do not have any data on $\mathrm{CKMB}$ levels in muscle tissue, but only systemic levels, we cannot draw any final conclusions on muscle involvement as reflected by systemic CKMB expression.

Another key component of skeletal muscles is Troponin-1 which controls striated muscle contraction and relaxation. Troponin-1 interacts with all major regulatory proteins in the sarcomeric thin filaments of cardiac and skeletal muscles [37]. Previous studies have shown very promising results regarding the use of slow skeletal Troponin I as a systemic biomarker for various muscle disorders [38] [39]. However, in the present study the slow skeletal Troponin I expression showed no differences between the 3 groups at the systemic level, but when measured locally in the muscle tissue the present study reveals an increased troponin-1 expression in stroke survivors when compared to healthy controls and individuals with $\mathrm{CP}$, while there was no difference between the $\mathrm{CP}$ and the healthy Ctrl groups (Figure 4). This finding is somewhat in contrast to other previous findings showing that subjects with spastic $\mathrm{CP}$ had less type I fibers and an increased amount of type IIX fibers when compared to healthy controls [40]. Another study showed that denervation through Botulinum toxin A injections caused a type 1 fiber loss and type 2 fiber predominance in the gastrochnemius muscle of children with CP [41]. However, we suggest that the increased gene expression of troponin-1 observed in the ST group might reflect a compensatory mechanism of the muscle, maybe as an effort to counteract the loss of Type I fibers due to denervation of the muscle after a CNS injury that has been acquired later in life. Unfortunately we can only speculate on this without drawing any final conclusions since no further data on fiber-types are available from the present participants.

CRP is an important component of the innate immune system and is known to increase in injured tissue [42]. The role of CRP within the innate immune system makes it a marker for inflammation. A 2003 study found that post-stroke elevated CRP levels predicted further disease-related ischemic events, suggesting that stroke survivors may be at risk for another ischemic event [43]. This is consistent with our observation that the CRP levels were significantly increased in stroke survivors (late onset) but not in individuals with CP (early onset. This indicates that CRP is primarily of importance for CNS injuries acquired later in life. Furthermore, this finding does also indicate that the stroke survivors still are affected by their stroke even several years (Mean 3.6 years) after the incident. The high level of CRP in stroke may either indicate inflammation in response to injured tissue or low-grade systemic inflammation. Increased levels of inflammation in stroke survivors, however, do not explain phenotypic differences in muscle atrophy and muscle contracture development. 


\section{Summary}

In summary, the present study shows that individuals with CNS injuries acquired either early (CP) or later in life (stroke survivors) both present differences and similarities in systemic biomarkers and in gene expression levels in skeletal muscle.

\section{Acknowledgements}

We thank all participants for contributing to this study.

\section{Ethics Approval and Consent to Participate}

All participants gave informed consent and the protocol was approved by the Regional Ethics Committee for Copenhagen (H-4-2014-047). The protocol was in compliance with the Helsinki Declaration.

\section{Availability of Data and Material}

All data generated during this study are included in this published article.

\section{Funding}

This project was funded by the Danish Research Council (DFF-1333-00197), and the Elsass Foundation.

\section{Author Contributions}

All authors have contributed equally to this work, and have approved the final version of the manuscript. All authors are designated as authors and are qualified for authorship, and are all listed as authors.

\section{Conflicts of Interest}

The authors declare that they have no competing interests.

\section{References}

[1] Mathewson, M.A. and Lieber, R.L. (2015) Pathophysiology of Muscle Contractures in Cerebral Palsy. Physical Medicine and Rehabilitation Clinics of North America, 26, 57-67. https://doi.org/10.1016/j.pmr.2014.09.005

[2] O’Dwyer, N.J., Ada, L. and Neilson, P.D. (1996) Spasticity and Muscle Contracture Following Stroke. Brain, 119, 1737-1749. https://doi.org/10.1093/brain/119.5.1737

[3] Pingel, J., Bartels, E.M. and Nielsen, J.B. (2016) New Perspectives on the Development of Muscle Contractures Following Central Motor Lesions. The Journal of Physiology, 595, 1027-1038. https://doi.org/10.1113/JP272767

[4] Graham, H.K., Rosenbaum, P., Paneth, N., et al. (2016) Cerebral Palsy. Nature Reviews Disease Primers, 2, Article No. 15082. https://doi.org/10.1038/nrdp.2016.5

[5] Kalkman, B.M., Bar-On, L., O’Brien, T.D., et al. (2020) Stretching Interventions in Children with Cerebral Palsy: Why Are They Ineffective in Improving Muscle Function and How Can We Better Their Outcome? Frontiers in Physiology, 11, 131. 
https://doi.org/10.3389/fphys.2020.00131

[6] Gough, M. and Shortland, A.P. (2012) Could Muscle Deformity in Children with Spastic Cerebral Palsy Be Related to an Impairment of Muscle Growth and Altered Adaptation? Developmental Medicine and Child Neurology, 54, 495-499. https://doi.org/10.1111/j.1469-8749.2012.04229.x

[7] Smith, L.R., Lee, K.S., Ward, S.R., et al. (2011) Hamstring Contractures in Children with Spastic Cerebral Palsy Result from a Stiffer Extracellular Matrix and Increased in Vivo Sarcomere Length. The Journal of Physiology, 589, 2625-2639. https://doi.org/10.1113/jphysiol.2010.203364

[8] Tedroff, K., Lowing, K., Jacobson, D.N., et al. (2011) Does Loss of Spasticity Matter? A 10-Year Follow-Up after Selective Dorsal Rhizotomy in Cerebral Palsy. Developmental Medicine and Child Neurology, 53, 724-729. https://doi.org/10.1111/j.1469-8749.2011.03969.x

[9] Sharma, M., McFarlane, C., Kambadur, R., et al. (2015) Myostatin: Expanding Horizons. IUBMB Life, 67, 589-600. https://doi.org/10.1002/iub.1392

[10] Schuelke, M., Wagner, K.R., Stolz, L.E., et al. (2004) Myostatin Mutation Associated with Gross Muscle Hypertrophy in a Child. The New England Journal of Medicine, 350, 2682-2688. https://doi.org/10.1056/NEJMoa040933

[11] Barber, L., Hastings-Ison, T., Baker, R., et al. (2011) Medial Gastrocnemius Muscle Volume and Fascicle Length in Children Aged 2 to 5 Years with Cerebral Palsy. Developmental Medicine and Child Neurology, 53, 543-548. https://doi.org/10.1111/j.1469-8749.2011.03913.x

[12] Nozoe, M., Kanai, M., Kubo, H., et al. (2016) Changes in Quadriceps Muscle Thickness, Disease Severity, Nutritional Status, and C-Reactive Protein after Acute Stroke. Journal of Stroke \& Cerebrovascular Diseases, 25, 2470-2474. https://doi.org/10.1016/j.jstrokecerebrovasdis.2016.06.020

[13] Ada, L., O’Dwyer, N. and O’Neill, E. (2006) Relation between Spasticity, Weakness and Contracture of the Elbow Flexors and Upper Limb Activity after Stroke: An Observational Study. Disability and Rehabilitation, 28, 891-897. https://doi.org/10.1080/09638280500535165

[14] Geertsen, S.S., Kirk, H., Lorentzen, J., et al. (2015) Impaired Gait Function in Adults with Cerebral Palsy Is Associated with Reduced Rapid Force Generation and Increased Passive Stiffness. Clinical Neurophysiology, 126, 2320-2329.

https://doi.org/10.1016/j.clinph.2015.02.005

[15] English, C., McLennan, H., Thoirs, K., et al. (2010) Loss of Skeletal Muscle Mass after Stroke: A Systematic Review. International Journal of Stroke, 5, 395-402. https://doi.org/10.1111/j.1747-4949.2010.00467.x

[16] Braun, T. and Gautel, M. (2011) Transcriptional Mechanisms Regulating Skeletal Muscle Differentiation, Growth and Homeostasis. Nature Reviews Molecular Cell Biology, 12, 349-361. https://doi.org/10.1038/nrm3118

[17] Herskind, A., Ritterband-Rosenbaum, A., Willerslev-Olsen, M., et al. (2016) Muscle Growth Is Reduced in 15-Month-Old Children with Cerebral Palsy. Developmental Medicine and Child Neurology, 58, 485-491. https://doi.org/10.1111/dmcn.12950

[18] Durand, P., Couto, R.A., Isakov, R., et al. (2016) Botulinum Toxin and Muscle Atrophy: A Wanted or Unwanted Effect. Aesthetic Surgery Journal, 36, 482-487. https://doi.org/10.1093/asj/sjv208

[19] Gough, M. (2009) Does Botulinum Toxin Prevent or Promote Deformity in Children with Cerebral Palsy? Developmental Medicine and Child Neurology, 51, 89-90. https://doi.org/10.1111/j.1469-8749.2008.03247.x 
[20] Plaisier, E., Chen, Z., Gekeler, F., et al. (2010) Novel COL4A1 Mutations Associated with HANAC Syndrome: A Role for the Triple Helical CB3[IV] Domain. American Journal of Medical Genetics Part A, 152, 2550-2555. https://doi.org/10.1002/ajmg.a.33659

[21] Vajsar, J. and Schachter, H. (2006) Walker-Warburg Syndrome. Orphanet Journal of Rare Diseases, 1, 29. https://doi.org/10.1186/1750-1172-1-29

[22] Decio, A., Tonduti, D., Pichiecchio, A., et al. (2015) A Novel Mutation in COL4A1 Gene: A Possible Cause of Early Postnatal Cerebrovascular Events. American Journal of Medical Genetics Part A, 167, 810-815. https://doi.org/10.1002/ajmg.a.36907

[23] Giorgio, E., Vaula, G., Bosco, G., et al. (2015) Two Families with Novel Missense Mutations in COL4A1: When Diagnosis Can Be Missed. Journal of the Neurological Sciences, 352, 99-104. https://doi.org/10.1016/j.jns.2015.03.042

[24] Jeanne, M., Labelle-Dumais, C., Jorgensen, J., et al. (2012) COL4A2 Mutations Impair COL4A1 and COL4A2 Secretion and Cause Hemorrhagic Stroke. American Journal of Human Genetics, 90, 91-101. https://doi.org/10.1016/j.ajhg.2011.11.022

[25] Tonduti, D., Pichiecchio, A., La Piana, R., et al. (2012) COL4A1-Related Disease: Raised Creatine Kinase and Cerebral Calcification as Useful Pointers. Neuropediatrics, 43, 283-288. https://doi.org/10.1055/s-0032-1325116

[26] Bi, D., Wang, H., Shang, Q., et al. (2016) Association of COL4A1 Gene Polymorphisms with Cerebral Palsy in a Chinese Han Population. Clinical Genetics, 90, 149-155. https://doi.org/10.1111/cge.12723

[27] Alamowitch, S., Plaisier, E., Favrole, P., et al. (2009) Cerebrovascular Disease Related to COL4A1 Mutations in HANAC Syndrome. Neurology, 73, 1873-1882. https://doi.org/10.1212/WNL.0b013e3181c3fd12

[28] Harteman, J.C., Van Haastert, I.C., Liem, K.D., Stroink, H., Bierings, M.B., Huisman, A. and De Vries, L. (2012) Atypical Timing and Presentation of Periventricular Haemorrhagic Infarction in Preterm Infants: The Role of Thrombophilia. Developmental Medicine \& Child Neurology, 54, 140-147. https://doi.org/10.1111/j.1469-8749.2011.04135.x

[29] Cabaniss, C.D. (1990) Chapter 32 Creatine Kinase. In: Walker, H.K., Hall, W.D., et al., Eds., Clinical Methods. The History, Physical, and Laboratory Examinations, Butterworth Publishers, Boston.

[30] Suleiman, H.M., Aliyu, I.S., Abubakar, S.A., et al. (2017) Cardiac Troponin T and Creatine Kinase MB Fraction Levels among Patients with Acute Ischemic Stroke in Nigeria. Nigerian Journal of Clinical Practice, 20, 1618-1621.

[31] Myers, M.G., Norris, J.W., Hachinski, V.C., et al. (1982) Cardiac Sequelae of Acute Stroke. Stroke, 13, 838-842. https://doi.org/10.1161/01.STR.13.6.838

[32] Barber, M., Morton, J.J., Macfarlane, P.W., et al. (2007) Elevated Troponin Levels Are Associated with Sympathoadrenal Activation in Acute Ischaemic Stroke. Cerebrovascular Diseases (Basel, Switzerland), 23, 260-266. https://doi.org/10.1159/000098325

[33] Nie, J., Tong, T.K., George, K., et al. (2011) Resting and Post-Exercise Serum Biomarkers of Cardiac and Skeletal Muscle Damage in Adolescent Runners. Scandinavian Journal of Medicine \& Science in Sports, 21, 625-629. https://doi.org/10.1111/j.1600-0838.2010.01096.x

[34] Shave, R., George, K.P., Atkinson, G., et al. (2007) Exercise-Induced Cardiac Troponin T Release: A Meta-Analysis. Medicine and Science in Sports and Exercise, 39, 2099-2106. https://doi.org/10.1249/mss.0b013e318153ff78 
[35] Sutidze, M., Sulakvelidze, M., Kochiashvili, D., et al. (2006) Creatine Kinase MB, Cardiac Troponin T and Cardiac Troponin I as the Markers of Rhabdomyolysis in Chronic Hemodialysis Patients. Georgian Medical News, No. 132, 68-71.

[36] Schwartz, J.G., Prihoda, T.J., Stuckey, J.H., et al. (1988) Creatine Kinase MB in Cases of Skeletal Muscle Trauma. Clinical Chemistry, 34, 898-901.

https://doi.org/10.1093/clinchem/34.5.898

[37] Layland, J., Solaro, R.J. and Shah, A.M. (2005) Regulation of Cardiac Contractile Function by Troponin I Phosphorylation. Cardiovascular Research, 66, 12-21. https://doi.org/10.1016/j.cardiores.2004.12.022

[38] Simpson, J.A., Labugger, R., Collier, C., et al. (2005) Fast and Slow Skeletal Troponin I in Serum from Patients with Various Skeletal Muscle Disorders: A Pilot Study. Clinical Chemistry, 51, 966-972. https://doi.org/10.1373/clinchem.2004.042671

[39] Chapman, D.W., Simpson, J.A., Iscoe, S., et al. (2013) Changes in Serum Fast and Slow Skeletal Troponin I Concentration Following Maximal Eccentric Contractions. Journal of Science and Medicine in Sport, 16, 82-85. https://doi.org/10.1016/j.jsams.2012.05.006

[40] Ponten, E.M. and Stal, P.S. (2007) Decreased Capillarization and a Shift to Fast Myosin Heavy Chain IIx in the Biceps Brachii Muscle from Young Adults with Spastic Paresis. Journal of the Neurological Sciences, 253, 25-33. https://doi.org/10.1016/j.jns.2006.11.006

[41] Valentine, J., Stannage, K., Fabian, V., et al. (2016) Muscle Histopathology in Children with Spastic Cerebral Palsy Receiving Botulinum Toxin Type A. Muscle \& Nerve, 53, 407-414. https://doi.org/10.1002/mus.24763

[42] Ansar, W. and Ghosh, S. (2013) C-Reactive Protein and the Biology of Disease. Immunologic Research, 56, 131-142. https://doi.org/10.1007/s12026-013-8384-0

[43] Arenillas, J.F., Alvarez-Sabin, J., Molina, C.A., et al. (2003) C-Reactive Protein Predicts Further Ischemic Events in First-Ever Transient Ischemic Attack or Stroke Patients with Intracranial Large-Artery Occlusive Disease. Stroke, 34, 2463-2468. https://doi.org/10.1161/01.STR.0000089920.93927.A7 